\title{
A General Model for Temperatures of Heterogeneous Nucleation of Supercooled Water Droplets
}

\author{
W. E. BARDSLEY \\ Earth Sciences, University of Waikato, Hamilton, New Zealand \\ M. M. KHATEP \\ Department of Geography, University of Otago, Dunedin. New Zealand
}

(Manuscript received 23 May 1983, in final form 21 November 1983)

\begin{abstract}
Asymptotic extreme-value theory is used as the basis of a stochastic model which predicts aspects of the freezing behavior of supercooled water droplets. The model gives a general theoretical base to an earlier empirical function relating droplet volume to droplet freezing temperature. At the same time, the model indicates that the established empirical relation is only one of three possible functions linking droplet volume and temperature of freezing.

The three functions arise as a consequence of three possible extreme-value distributions of droplet freezing temperatures, and the form of each function predicts the type and parameter values of the distributions concerned. The proposed model is amenable to rigorous experimental verification since both the distribution type and parameter values can be estimated independently from droplet freezing data.
\end{abstract}

\section{1. 具踏oduction}

It is well known that small water droplets often freeze at temperatures below $0^{\circ} \mathrm{C}$. If the droplets are composed of pure water, freezing will only occur at some defined critical temperature-the temperature of homogeneous nucleation. Freezing temperatures are higher and more variable if the droplets contain small foreign particles, the initiation of the ice phase in this case being referred to as heterogeneous nucleation. It is the temperature of heterogeneous nucleation which is the concern of the present paper. As well as being of general scientific interest, the prediction of freezing temperature distributions is likely to be of advantage in the study of cloud seeding, which is often concerned with the induced nucleation of supercooled cloud droplets.

The exact nature of heterogeneous nucleation is still imperfectly known, and a variety of possible physical mechanisms have been suggested over the years (Rosinski, 1979). A more non-deterministic line of investigation was initiated when Langham and Mason (1958) demonstrated that some general characteristics of droplet freezing patterns could be predicted by a simple stochastic model, given certain restrictive assumptions.

This paper utilizes asymptotic extreme-value theory to obtain a more robust generalized version of the Langham-Mason freezing model. The general model has the advantage of avoiding arbitrary assumptions, while at the same time giving a greater degree of applicability to different freezing situations.

It is emphasized that the general model is stochastic in nature and is not tied to physical aspects of nucleation beyond that presented by Langham and Mason (1958). The main concern of this paper is to use the model to demonstrate that many of the observed characteristics of droplet freezing temperatures can be explained as simple stochastic behavior, regardless of the exact nature of the nucleation process at the molecular level.

\section{The Langhasm-Mason freezing model}

The Langham-Mason model views the foreign particles within the droplets as temperature-dependent ice nuclei. In other words, each particle is seen to be associated with some specific "activation temperature" at which that particle will initiate ice formation. Of course, the number of ice nuclei in any given droplet is a constant unrelated to droplet temperature.

Once freezing has been initiated in a supercooled droplet, the freezing process spreads almost instantaneously through the droplet. Given this rapid transformation, the Langham-Mason model interprets the freezing temperature of a given droplet as being equivalent to the highest activation temperature of the contained particles. Symbolically

$$
X=\max \left(Y_{1}, Y_{2}, Y_{3}, \ldots, Y_{N}\right),
$$


where $X$ is a random variable denoting the droplet freezing temperature, $Y$ the activation temperature of the $i$ th contained particle, and $N$ is the number of ice nuclei in the droplet.

By assuming an exponential distribution of activation temperatures, Langham and Mason (1958) were able to derive the well-known empirical volume-freezing relation (Bigg, 1953)

$$
x(0.5)_{V}=B \ln V+A
$$

where $x(0.5)_{V}$ is the median freezing temperature in ${ }^{\circ} \mathrm{C}$ of droplets of constant volume $V$. The constants $A$ and $B$ are dependent upon the nature and concentration of particles in the water mass from which the droplets were created.

An obvious restriction of the above model is the requirement of an explicit distribution of $Y$ in order to obtain the relation (2). In fact, distributions of activation temperatures are more likely to be multimodal because of mixtures of different types of foreign particles. In the general model, the natural extension is made to the limit distribution of $X$ for large numbers of contained particles. This procedure permits the derivation of (2) and yields two other volume-freezing relations, without requiring knowledge of the distribution of $Y$. The new model is therefore "general," although it will not be applicable when only a few ice nuclei are present per droplet. With such small numbers of nuclei, the distributions of droplet freezing temperatures may have complex multimodal forms reflecting the distribution of $Y$.

\section{Basis of the general model}

From a statistical viewpoint, it is evident from (1) that the Langham-Mason model is concerned with the largest order statistic (highest nucleation temperature, droplet freezing temperature) from a random sample of nucleation temperatures. The sample size for any one droplet thus corresponds to the number of nuclei contained within that droplet.

Since droplet freezing temperatures can be interpreted as largest order statistics, predictions can be made of aspects of droplet freezing using known mathematical properties of order statistics. The situation is slightly complicated by the fact that the sample size is a random variable. However, given the dispersed nature of the nuclei, it can be reasonably assumed that the number of nuclei per droplet of (constant) volume $V$ will be a Poisson random variable. The required mathematical information therefore relates to the particular case of order statistics with Poisson-distributed sample sizes.

The requirement for a large number of nuclei can now be expressed more explicitly as a large value of $\lambda_{V}$, where $\lambda_{V}$ denotes the Poisson parameter, corresponding to the average number of nuclei per droplet in a set of droplets, all of volume $V$. It is assumed that any droplet "set" has been created from the same water source. Droplets from different water sources will of course possess different values of $\lambda_{V}$.

From the above discussion, the expression (1) in the Langham-Mason model can be defined in terms of the general model as

$$
X=\max \left(Y_{1}, Y_{2}, Y_{3}, \ldots, Y_{K}\right),
$$

where $K$ is a Poisson random variable with (large) parameter value $\lambda_{V}$.

Considerations of the distribution of $X$ in (3) come under the general heading of asymptotic extreme-value theory. The theory was originally derived with respect to constant sample size but was later extended to include the case of Poisson-distributed sample sizes. Although aspects of the asymptotic theory have been widely applied in the analysis of floods and extreme climatic events, it would be helpful at this point to list the features of the theory which are relevant to the general model.

In terms of the present symbolism, the asymptotic theory states that, under very general conditions, the random variable $X$ in (3) will follow one of only three possible types of distribution, regardless of the distribution of the random variable $Y$. The derivation of these three extreme-value distributions was originally carried out by Fisher and Tippett (1928) and Gnedenko (1943). The extension to Poisson sample size is discussed by Epstein (1949) and Bardsley and Manly (1979). Following Johnson and Kotz (1970), the distribution functions of the three types can be written

Type 1:

$$
\operatorname{pr}(X \leqslant x)=\exp \{-\exp [-(x-\xi) / \theta]\},
$$

Type 2:

$$
\operatorname{pr}(X \leqslant x)=\exp \left\{-[(x-\omega) / \delta]^{-\beta}\right\}, \quad x \geqslant \omega,
$$

Type 3:

$$
\operatorname{pr}(X \leqslant x)=\exp \left\{-[(\epsilon-x) / \gamma]^{\alpha}\right\}, \quad x \leqslant \epsilon,
$$

where $\xi, \omega$ and $\epsilon$ are location parameters and $\theta, \delta$ and $\gamma$ are scale parameters and $\alpha, \beta$ are shape parameters. The term $\operatorname{pr}(X \leqslant x)$ denotes the probability that the random variable $X$ will be less than some specified value $x$, where $x$ will be taken as ${ }^{\circ} \mathrm{C}$. In physical terms, $\operatorname{pr}(X \leqslant x)$ corresponds to the proportion of a (large) droplet set which is still unfrozen at temperature $x$.

The probability density functions of the three distribution types are shown in Fig. 1, for selected parameter values. A negative $x$ axis is used in this case since we will be concerned with measurements on a negative temperature scale. The three distributions are all quite simple in that they never possess more than a single mode, but Types 2 and 3 may still show considerable variety of form. The Type 1 (Gumbel) distributions possess only location and scale parameters and so are shape-invariant. 

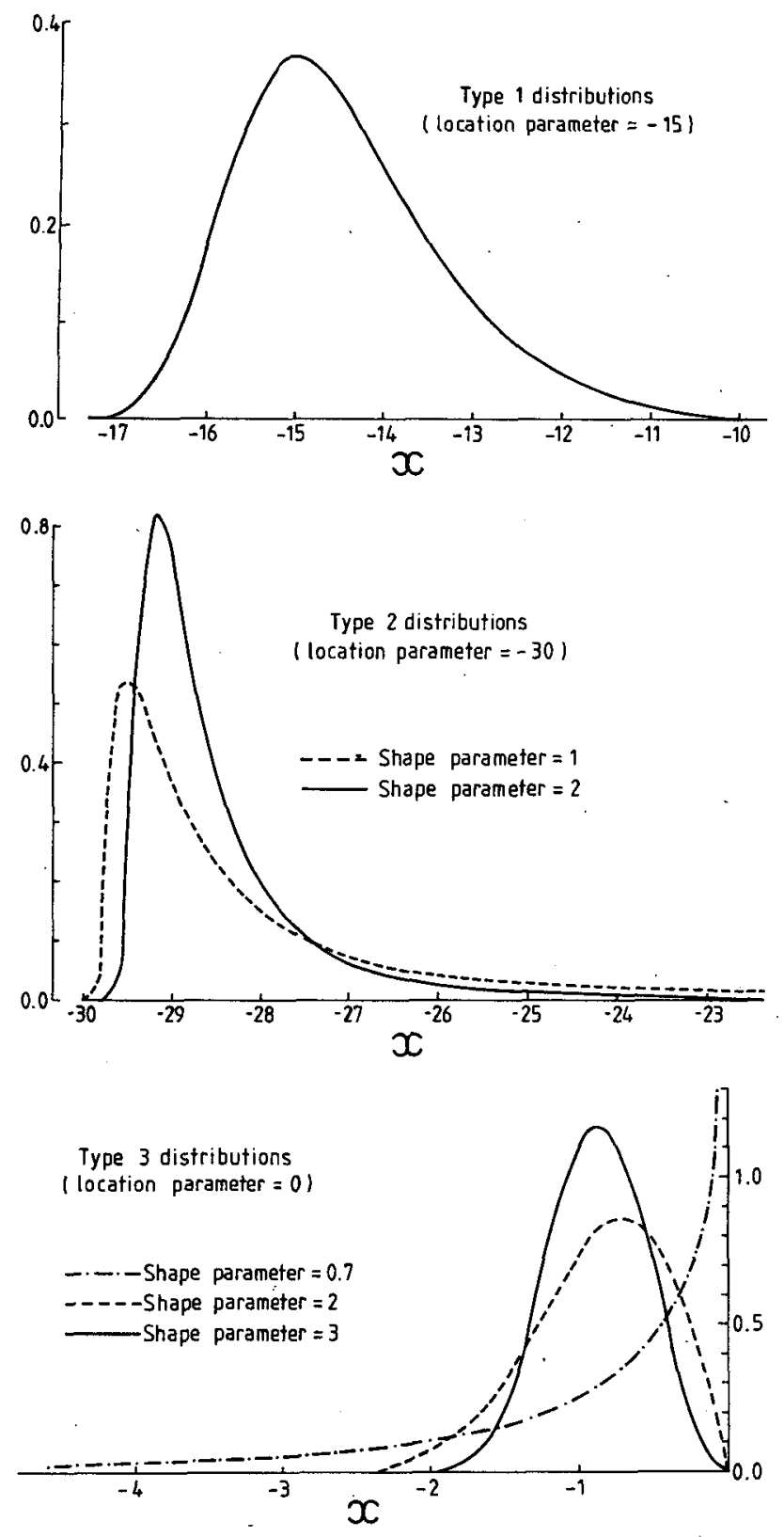

FIG. 1. Extreme value density functions for selected parameters (probability density on vertical scale).

Although the asymptotic theory indicates that one of the extreme value distributions should always arise for sufficiently large $\lambda_{V}$, it is not possible to predict which of the three types will apply without some knowledge of the distribution of $Y$. Type 3 distributions arise when the distribution of $X$ is influenced by the presence of an upper bound on $Y$. The other two distributions occur in the absence of an effective upper bound, the form of the upper tail of the distribution of $Y$ determining which of the two types will apply.

Since $0^{\circ} \mathrm{C}$ represents an obvious upper bound to $Y$,
Type 3 distributions are most likely to arise when droplets freeze at higher temperatures. The presence of efficient ice nuclei is likely to cause $\epsilon$ to be less than $0^{\circ} \mathrm{C}$, the exact value of this "threshold parameter" being determined by the nature of the nuclei type concerned. The occurrence of Type 2 distributions is less simple to predict in the context of droplet freezing. However, the Type 2 location parameter is likely to correspond to the temperature of homogeneous nucleation, suggesting that these distributions may be associated with droplets freezing at low temperatures. When they arise, the simple Type 1 distributions will be located at intermediate freezing temperatures such that the distribution tails will not be influenced by either the upper or lower bounds to temperatures of heterogeneous nucleation.

From the viewpoint of deriving volume freezingtemperature relations, it is of importance to know the relation between the freezing distribution parameter values and the magnitude of $\lambda_{V}$. Fortunately, this relation is simply the well-known "reproductive property" of extreme value distributions. In effect, the reproductive property states that if $\lambda_{V}$ is increased further beyond an already large value, the type of extreme value distribution of $X$ will remain unchanged. Furthermore, the only parameters which vary are $\xi, \delta$, and $\gamma$ for distribution types 1,2 , and 3 respectively. In other words, increasing $\lambda_{V}$ as a consequence of increasing $V$ will alter the freezing distribution by location and/or scale, but the shape remains unaltered. The three varying parameters are linked to $\lambda_{V}$ by simple mathematical relations, summarized in Table 1 where the parameters concerned are expressed as functions of $\lambda_{V}$. Further discussion of the reproductive property is given by Gumbel $(1958,159-161)$.

As suggested by the relations in Table 1, each of the three possible extreme-value types generates a unique mathematical function linking droplet volume and the quantiles of the associated distributions of droplet freezing temperatures. In the following three sections, the relevant function is derived for each of the distribution types.

\section{Type 1 distributions}

From the inverse of (4), any specified quantile of a Type 1 freezing distribution for constant-volume droplets is given by

TABLE 1. The extreme-value reproductive property in droplet freezing: varying parameters as functions of $\lambda_{V} ; a, d$, and $h$ are constants for a given water source.

\begin{tabular}{ccc}
\hline Distribution & $\begin{array}{c}\text { Varying } \\
\text { parameter }\end{array}$ & $\begin{array}{c}\text { Parameter as } \\
\text { a function of } \lambda_{V}\end{array}$ \\
\hline Type 1 & $\xi$ & $\begin{array}{l}a+\theta \lambda_{V} \\
d \lambda_{V}^{1 / \beta} \\
\text { Type 2 }\end{array}$ \\
Type 3 & $\delta$ & $h \lambda_{V}^{1 / \alpha}$ \\
\hline
\end{tabular}




$$
x(p)_{V}=\xi_{V}-\theta \ln \ln p^{-1},
$$

where $V$ denotes droplet volume. The quantile $x(p)_{V}$ corresponds to the median for the particular case of $p=0.5$. The parameter $\theta$ is not subscripted here since its value is independent of $\lambda_{V}$ and therefore of $V$.

From the Type 1 reproductive property (Table 1), increasing $\lambda_{V}$ as a consequence of increasing the droplet size will result in $\xi_{V}$ increasing with $V$ as

$$
\begin{aligned}
\xi_{V} & =a+\theta \ln \lambda_{V} \\
& =a+\theta \ln \lambda_{1}+\theta \ln V,
\end{aligned}
$$

where $a$ and $\theta$ are constants for the water sample concerned, and $\lambda_{1}$ is the average number of particles per unit volume. Substituting for $\xi_{V}$ in (7) gives the desired quantile/volume relation

$$
x(p)_{V}=\theta \ln V+a+\theta\left(\ln \lambda_{1}-\ln \ln p^{-1}\right)
$$

from which (2) is obtained as a special case by setting $p$ to 0.5 and gathering constants.

It will be noted that the derivation of $(9)$ required only $\lambda_{V}$ to be large, and the distribution of $Y$ to be of the very general exponential type (Gumbel, 1958, 120). The frequent appearance of the relation (2) in experimental data can therefore be explained in a more realistic way than the ubiquitous exponential distribution of activation temperatures required by the Langham-Mason model.

\section{Type 2 distributions}

From the inverse of (5), any specified quantile of a Type 2 distribution of freezing temperatures for droplets of volume $V$ is given by

$$
x(p)_{V}=\omega_{V}+\delta_{V}(-\ln p)^{-1 / \beta} .
$$

The parameter $\omega_{V}$ is subscripted with respect to $V$ in this case, since this lower bound may itself vary as a function of volume. This situation would apply, e.g., if $\omega_{V}$ corresponded to the temperature of homogeneous nucleation.

Using similar arguments as in the Type 1 case, it follows from the reproductive property that increasing $\lambda_{V}$ as a consequence of increasing droplet volume will result in $\delta_{V}$ increasing with volume as

$$
\delta_{V}=b V^{1 / \beta} \text {, }
$$

where the various constants have been gathered into the single positive constant $b$, the value of $b$ depending on the nature of the parent water of the droplets. At the same time, $\beta$ remains unaltered by any volume change. The required quantile-volume relation for the Type 2 case is therefore obtained as

$$
x(p)_{V}=\omega_{V}+b V^{1 / \beta}(-\ln p)^{-1 / \beta},
$$

and taking logs gives the linear function of $\ln V$

$$
\ln \left[x(p)_{V}-\omega_{V}\right]=\beta^{-1} \ln V+\ln [b(-\ln p)]^{-1 / \beta} .
$$

The expression (13) is the Type 2 equivalent of (9) and represents a new theoretical volume-temperature relation which can be checked against freezing data.

\section{Type 3 distributions}

Proceeding as in the other two cases, the inverse of (6) yields any specified quantile of a Type 3 distribution of droplet freezing temperatures as

$$
x(p)_{V}=\epsilon-\gamma_{V}(-\ln p)^{1 / \alpha} .
$$

Utilizing the Type 3 reproductive property (Table 1), increasing $\lambda_{V}$ as a consequence of increasing droplet volume will result in $\gamma_{V}$ decreasing with volume as

$$
\gamma_{V}=c V^{-1 / \alpha} \text {, }
$$

where $c$ is a positive constant for a given water sample. The shape parameter $\alpha$ is unaffected by volume change. Substituting for $\gamma_{V}$ in (14) gives the Type 3 quantilevolume relation

$$
x(p)_{V}=\epsilon-c V^{-1 / \alpha}(-\ln p)^{1 / \alpha}
$$

and taking logs gives the linear function of $\ln V$

$$
\ln \left[\epsilon-x(p)_{V}\right]=-\alpha^{-1} \ln V+\ln \left[c(-\ln p) \alpha^{-1}\right] .
$$

Again, (17) represents a predicted relation which is new in droplet freezing studies.

\section{Variable droplet size}

All the expressions presented in this paper have been with respect to the case where all the droplets in a given set have the same volume. This constant volume basis was required to justify Poisson-distributed particle numbers (per droplet), thus permitting the use of asymptotic extreme-value theory for large $\lambda_{V}$.

If the droplet size is a random variable (of unknown distribution), then $\lambda_{V}$ also becomes a random variable and the particle number per droplet will follow some unknown compound Poisson distribution. In this situation, a large average number of particles per droplet is no longer a sufficient condition to justify application of the asymptotic theory. However, droplet freezing temperatures can still be predicted to follow extreme value distributions given 1) a large average number of particles per droplet, and 2) an average droplet volume which is large in relation to the mean deviation of the droplet volumes (Bardsley and Manly, 1979). Given 1) and 2), (9), (13) and (17) will hold as before, but with $V$ replaced by $E(V)$, the average value of $V$.

\section{Comparison with observations}

The general model of droplet freezing gives rise to a number of specific predictions which can be tested against experimental data. However, a complete verification of the model is beyond the scope of the present paper and is best left to those in the field with direct access to the raw data. We confine ourselves here to 
pointing out some observations from the literature which are in accordance with the model, and offer some suggestions as to how the model might be most simply tested.

Leaving aside the three quantile-volume relations, the most fundamental prediction of the model is that, given large numbers of nuclei, droplet freezing temperatures should follow extreme-value distributions. On the basis of the frequently-reported occurrence of the empirical relation (2), the prediction can be made that the Type 1 distributions are likely to be the most common. Some indirect evidence for Type 1 distributions can be inferred from some results given by Vali and Stansbury (1966). They noted that in-some freezing experiments, the instantaneous rate of freezing increased exponentially with decreasing temperature. A freezing rate relation of this form is predicted by the general model whenever the droplet freezing temperatures follow Type 1 extreme-value distributionsas can be verified from the exponential nature in Type 1 distributions of the ratio: density function/cumulative distribution function. (In physical terms, the height of any density function at a specified temperature is proportional to the number of droplets which freeze at that temperature, while the distribution function is proportional to the number of droplets still remaining unfrozen at that temperature).

Some recent results obtained by Montefinale $e t$ al. (1976) can be interpreted in terms of the presence of Type 3 freezing distributions. In particular, these workers observed that when $-x$ was raised to a suitable power, it formed a linear relation with the $\log$ of the proportion of droplets unfrozen at that temperature. This linear form is predicted by the general model whenever the droplet freezing temperatures follow a Type 3 distribution with $\epsilon=0$. This can be verified from (6), which gives the proportion of droplets unfrozen at temperature $x$ for the Type 3 case. It is evident that the $\log$ of this proportion will plot as a linear function of $(-x)^{\alpha}$, with slope $-\gamma^{-\alpha}$. The linearity of the absolute freezing temperature raised to a power holds only for the special case of $\epsilon=0$. However, linearity of experimental data would hold to a first approximation provided the threshold temperature was not too far below $0^{\circ} \mathrm{C}$, as was the case with the data of Montefinale $e t$ al.

As noted earlier, Type 3 distributions are most likely to come into effect at higher freezing temperatures when the upper bound to freezing begins to have an influence on the droplet freezing distribution. In this regard, Pitter and Pruppacher (1973) made the interesting observation that (2) did not hold at higher freezing temperatures. While this in itself is not evidence for a Type 3 distribution, the observation does indicate that some freezing distribution other than the Type 1 must be involved.

There appears to be no immediate evidence for the existence of Type 2 freezing distributions. However, it would be of interest to check this distribution against observations obtained from droplets freezing near the temperature of homogeneous nucleation.

A useful feature of the extreme-value distributions is that they are amenable to simple graphical tests. The technique is standard in extreme-value applications but the method is briefly outlined here since it may be unfamiliar to those engaged in the collection of freezing data. If $G_{X}$ is the sample proportion of droplets unfrozen at a droplet freezing temperature $X$, then from (4), (5) and (6) the quantity $-\ln \left(-\ln G_{X}\right)$ will plot a linear function of $X, \ln (X-\omega)$ or $\ln (\epsilon-X)$ for types 1, 2 and 3, respectively. Given the underlying distribution, the only departure from linearity arises from sampling variation of $G_{X}$.

The graphical plotting procedure is illustrated in Fig. 2 with respect to two frequency distributions of freezing temperatures. The histogram of Fig. 2a was obtained from the freezing of droplets created from rainwater (Vali, 1971), while the lower distribution was generated by the freezing of droplets of distilled water (Vali and Stansbury, 1966). The rather jagged appearance of the rainwater histogram is due in this case to a relatively small number of droplets, not the lesser purity of the water. If there is any relation to water quality, the general model will be more applicable to environmental water because of the higher value of $\lambda_{V}$.

Because the data of the histograms is already grouped, the $X$ values were taken as the class midpoints, and $G_{X}$ as the proportion of area to the left of the individual midpoints. The negative skewness of the rainwater distribution suggests the Type 3 family as the most likely candidate of the three possibilities. After some trial values of $\epsilon$, a reasonable degree of linearity was obtained in the $\log$ plot with $\epsilon=-6.2^{\circ} \mathrm{C}$, indicating compatibility of the data with an underlying Type 3 distribution displaced to this threshold value. The greater number of droplets associated with Fig. 2b brings out the underlying distribution more clearly. In this case, the distribution appears to be of the Type 1 form, a conclusion further supported by the high degree of linearity of the corresponding log plot.

Apart from the graphical methods, a number of estimators have been developed for use with extremevalue distributions. Techniques developed for the threeparameter Weibull distribution are directly applicable to the Type 3 case because of the simple relation between these two distributions (Wyckoff et al., 1980). A test for distinguishing between the three extremevalue types is given by Otten and Van Montfort (1978).

Although a study of observed freezing distributions provides some check of the general model, the most rigorous test is achieved by using the three predicted quantile-volume relations (9), (13) and (17). This arises from the fact that these relations predict not only the type of freezing distribution but also some of the parameters of the individual distributions. The observed 

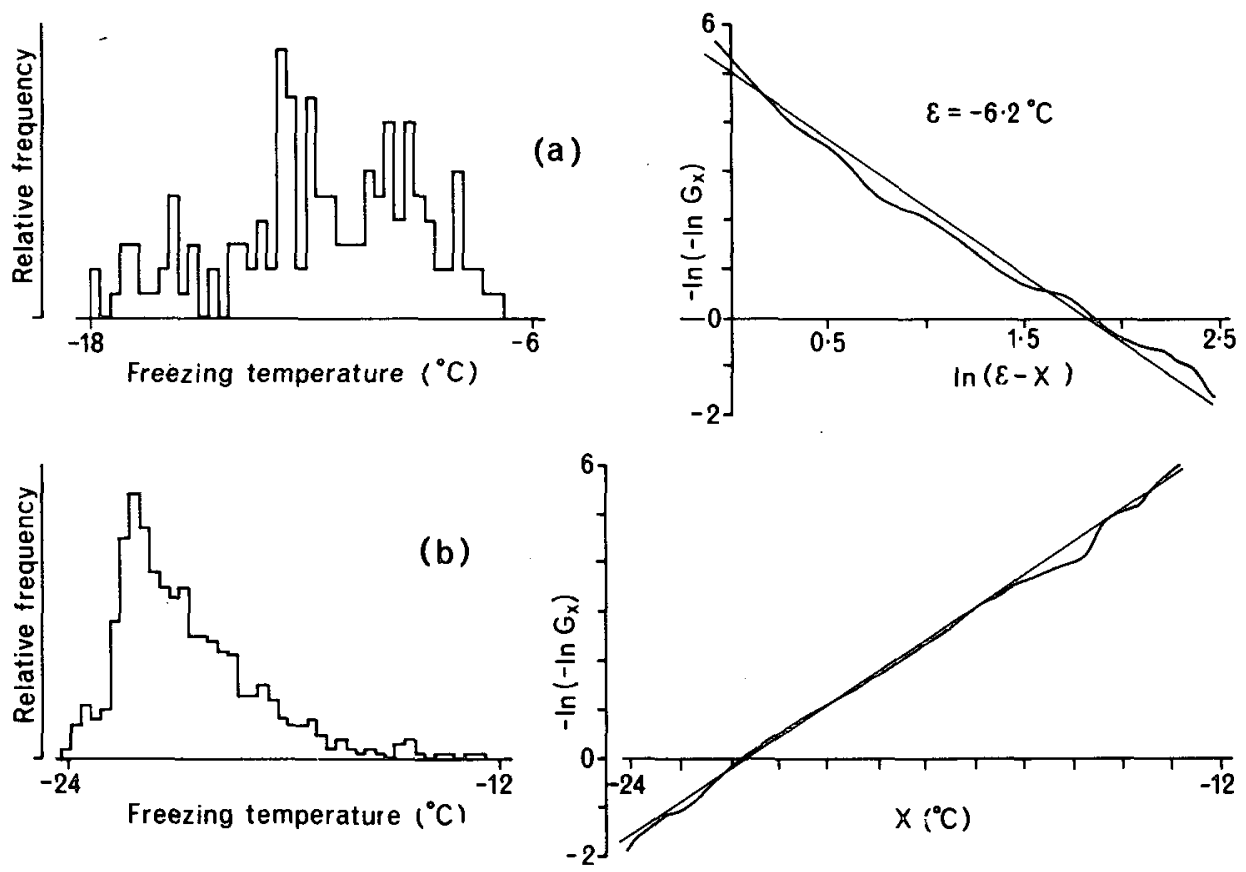

FIG. 2. Observed distributions of droplet freezing temperatures and associated log plots. The distributions of (a) and (b) after Vali (1971) and Vali and Stansbury (1966), respectively.

freezing distributions can then be compared with those predicted from the quantile-volume relation as estimated, say, from the observed median freezing temperature of droplet sets. For example, if a linear relation is revealed by a plot of $x(0.5)_{V}$ against $\ln V$, then the distributions of droplet freezing temperatures for all volumes should be Type 1 with a common scale parameter $\theta$. This common value of $\theta$ is predicted from the gradient of the linear function. Using this value of $\hat{\theta}$, the value of the location parameter $\xi_{V}$ is predicted for a given freezing distribution from the expression for the median of a Type 1 distribution

$$
x(0.5)_{V}=\xi_{V}-\theta \ln \ln 2,
$$

where $x(0.5)_{V}$ would be read from the linear function concerned.

Applying the above procedure to the specific linear function graphed by Bigg (1953), the slope with respect to $\ln V$ is obtained as $1.0^{\circ} \mathrm{C}$. Reading from Bigg's graph, the value of $x(0.5)_{V}$ for droplets of $1 \mathrm{~mm}$ diameter is obtained as $-23.7^{\circ} \mathrm{C}$, and $\xi_{V}$ is thus approximately $-24^{\circ} \mathrm{C}$. The predicted distribution is shown in Fig. 3, superimposed on the empirical distribution obtained from the freezing data of over $10001 \mathrm{~mm}$ droplets (Bigg, 1953). Although the overlap is not perfect, the similarity is striking when it is remembered that both the type and parameter values of the predicted distribution were obtained independently of the individual droplet freezing temperatures.

It is evident from the linear functions (13) and (17) that a similar type of approach may be taken with respect to the Type 2 and Type 3 cases. Since (13) and (17) are entirely new functions in the field of droplet freezing, experimental confirmation of their existence would provide strong support for the general model.

Finally, it should be mentioned that experimental verification can be carried out using sample means as well as sample quantiles. This arises from the fact that changing the droplet volume does not alter the shape of the extreme value freezing distributions (Table 1 ). The true means of a volume sequence of freezing distributions therefore correspond to some fixed quantile, so the sample mean in effect estimates a quantile value. In fact, it may be more desirable to use the sample

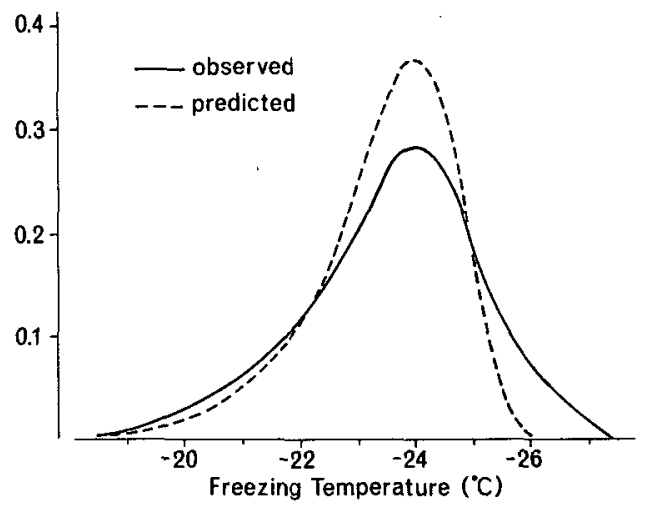

FIG. 3. Observed and predicted probability distributions of droplet freezing (probability density on vertical scale). Observed distribution after Bigg (1953). 
mean rather than the median in order to minimize the effect of sampling variation. On the other hand, it is well known that sample means may be sensitive to outlying observations.

\section{Conclusion}

Extreme-value theory provides a unifying framework for the analysis of the freezing behavior of supercooled water droplets. The general model presented here is consistent with many of the observations of droplet freezing, and a number of predictions are made which can be readily tested by further experimentation.

It should also be recognized, however, that the existence of the general model would imply some clear limitations on the information that could be extracted from the data of droplet freezing. In particular, given that the droplets contain large numbers of ice nuclei, it will not be possible to work backwards and deduce the activation distribution using the freezing distributions of droplets of different volumes. This arises from the extreme value property that for $\lambda_{V}$ large, the distribution of $X$ does not uniquely determine the distribution of $Y$, regardless of the number of different sets of droplet volumes. On the other hand, the "different volume" approach will be a useful technique provided there are not too many ice nuclei present. In practice, low nuclei numbers can always be achieved by dilution with pure water, as described by Schnell and Vali (1976).

With respect to further generalization of the model, it may be possible to allow for a secondary effect resulting from freezing initiated by time-varying clusters of water molecules in icelike configurations. The existence of this process is suggested by the observation that water droplets held at a constant temperature will sometimes freeze well after this temperature has been reached (Vali and Stansbury, 1966). An extreme-value approach should also be applicable here, since droplet freezing is initiated if the largest molecular cluster exceeds some critical magnitude.

\section{REFERENCES}

Bardsley, W. E., and B. F. J. Manly, 1979: Note on the distribution. of flood maxima for random sample size. J. Hydrol., 42, 187-. 193.

Bigg, E. K., 1953: The supercooling of water. Proc. Phys. Soc. London, B66, 688-694.

Epstein, B., 1949: A modified extreme value problem. Ann. Math. Statist., 20, 99-103.

Fisher, R. A., and L. H. C. Tippett, 1928: Limiting forms of the frequency distribution of the largest or smallest member of a sample. Proc. Cambridge Phil. Soc., 24, 180-190.

Gnedenko, B., 1943: Sur la distribution limite du terme maximum d'une série aléatoire. Ann. Math., 44, 423-453.

Gumbel, E. J., 1958: Statistics of Extremes. Columbia University Press, 372 pp.

Johnson, N. L., and S. Kotz, 1970: Continuous Univariate Distributions, Vol. 1. Wiley \& Sons, $300 \mathrm{pp}$.

Langham, E. J., and B. J. Mason, 1958: The heterogeneous and homogeneous nucleation of supercooled water. Proc. Roy. Soc. London, A247, 493-504.

Montefinale, T., A. C. Montefinale and H. M. Papee, 1976: Analysis of ice-nucleation thresholds of water-insoluble solids. J. Colloid Interface Sci., 54, 409-414.

Otten, A., and M. A. J. Van Montfort, 1978: The power of two tests on the type of distributions of extremes. $J$. Hydrol., 37, 195199.

Pitter, R. L., and H. R. Pruppacher, 1973: A wind tunnel investigation of freezing of small water drops falling at terminal velocity in air. Quart. J. Roy. Meteor. Soc., 99, 540-550.

Rosinski, J., 1979: The role of natural and man-made ice-forming nuclei in the atmosphere. Adv. Colloid Interface Sci., 10, 315367.

Schnell, R. C., and G. Vali, 1976: Biogenic ice nuclei. Part I: Terrestrial and marine sources. J. Atmos. Sci., 33, 1554-1564.

Vali, G., 1971: Quantitative evaluation of experimental results on the heterogeneous freezing nucleation of supercooled liquids. J. Atmos. Sci., 28, 410-415.

the heterogeneous nucleation of ice. Can. J. Phys., 44, 477502 .

Wyckoff, J., L. J. Bain and M. Engelhardt, 1980: Some complete and censored sampling results for the three-parameter Weibull distribution. J. Statist. Comp. Simul., 11, 139-151. 